\title{
Fatores de Previsão de Hipotensão Arterial Precoce em Anestesia Subaracnóidea *
}

\section{Predictors of Early Hypotension During Spinal Anesthesia}

Getúlio Rodrigues de Oliveira Filho, TSA ${ }^{1}$, Jorge Hamilton Soares Garcia, TSA ${ }^{2}$, Ranulfo Goldschimidt, TSA ${ }^{2}$, Adilson José Dal Mago, TSA ${ }^{2}$, Marcos Aguiar Cordeiro ${ }^{3}$, Felipe Ceccato ${ }^{3}$

\section{RESUMO}

Oliveira Filho GR, Garcia JHS, Goldschimidt R, Dal Mago AJ, Cordeiro MA, Ceccato F - Fatores de Previsão de Hipotensão Arterial Precoce em Anestesia Subaracnóidea

Justificativa e Objetivos - A identificação de fatores de previsão de hipotensão arterial durante bloqueios subaracnóideos pode interferir na escolha da técnica ou na utilização de medidas preventivas. Este estudo avaliou fatores pré-anestésicos como previsores independentes de hipotensão arterial durante bloqueio subaracnóideo.

Método - Foram estudados 76 pacientes de ambos os sexos, submetidos à anestesia subaracnóidea com bupivacaína 0,5\% hiperbárica. Foram coletados: idade, sexo, peso, altura, índice de massa corporal, história de hipertensão arterial, uso de drogas anti-hipertensivas, medicação pré-anestésica, pressão arterial sistólica (PAS) e diastólica (PAD) no braço e no tornozelo, freqüência cardíaca (FC), pressão de pulso arterial, indice de sobrecarga vascular, índice tornozelo/braço de PAS, doses de bupivacaína, nível superior do bloqueio sensitivo, e a menor PAS, medida a intervalos de 2,5 minutos até o vigésimo minuto. Oxigênio a 3 L. $\mathrm{min}^{-1}$ foi administrado, segundo sorteio. Hipotensão arterial foi definida como redução da PAS a valores inferiores a $80 \%$ do nível pré-anestésico ou PAS menor que 90 $\mathrm{mmHg}$. Foi utilizada regressão logística para identificar as variáveis associadas com a ocorrência de hipotensão arterial.

Resultados - Foram fatores de previsão de hipotensão arterial: idade maior que 45 anos, sexo feminino e nível superior do bloqueio sensitivo acima de T7.

Conclusões - Foram identificados fatores de previsão independentes de redução tensional sistólica acima de $20 \%$ dos valores pré-anestésicos: a idade acima de 45 anos, o sexo feminino e o nível superior do bloqueio sensitivo acima de T7.

UNITERMOS: COMPLICAÇÕES, hipotensão arterial; TÉCNICAS ANESTÉSICAS, Regional: subaracnóidea

\footnotetext{
* Recebido do (Received from) CET/SBA Integrado de Anestesiologia da SES-SC, Florianópolis, SC

1. Responsável pelo CET/SBA do SES-SC

2. Instrutor do CET/SBA do SES-SC

3. $M E_{2}$ do CET/SBA do SES-SC

4. $M E_{1}$ do CET/SBA do SES-SC
}

Apresentado (Submitted) em 04 de dezembro de 2000

Aceito (Accepted) para publicação em 09 de janeiro de 2001

Correspondência para (Mail to):

Dr. Getúlio Rodrigues. de Oliveira Filho

Rua Luiz Delfino 111/902

88015-360 - Florianópolis, SC

E-mail: grof@th.com.br

\section{SUMMARY}

Oliveira Filho GR, Garcia JHS, Goldschimidt R, Dal Mago AJ, Cordeiro MA, Ceccato F - Predictors of Early Hypotension During Spinal Anesthesia

Background and Objectives - The identification of hypotension predicting factors during spinal anesthesia may interfere in the choice of the technique or call for preventive measures. This study aimed at identifying pre-anesthetic factors as independent hypotension predictors during spinal anesthesia. Methods - Participated in this study 76 adult patients of both genders undergoing spinal anesthesia with $0.5 \%$ hyperbaric bupivacaine. The following parameters were collected: age, gender, weight, height, body mass index, systemic hypertension history, use of anti-hypertensive agents, preanesthetic medication, ankle and arm systolic and diastolic blood pressure, heart rate, pulse pressure, vascular overload, ankle-arm SBP index, bupivacaine doses, upper sensory block level and the lowest SBP measured at 2.5 minute intervals for 20 minutes. Patients were randomly allocated to receive oxygen $\left(3{\mathrm{~L} . \mathrm{min}^{-1}}^{-1}\right.$. Hypotension was defined as systolic blood pressure below $80 \%$ of control or below $90 \mathrm{mmHg}$. Logistic regression was applied to identify independent hypotension predictors.

Results - The following variables were independently associated to systolic hypotension: more than 45 years of age, female gender and upper sensory level above T7.

Conclusions - More than 45 years of age, female gender and upper sensory level above T7 were identified as independent predictors of early hypotension during spinal anesthesia.

KEY WORDS: ANESTHETIC TECHNIQUES, Regional: spinal block; COMPLICATIONS, arterial hypotension

\section{INTRODUÇÃO}

$\Delta$ identificação de pacientes com maior risco de desenvolAver hipotensão durante raquianestesia já foi objeto de estudo, do qual resultou um modelo logístico que incluiu o nível sensitivo acima de $T 6$ e a idade acima de 50 anos como previsores independentes de hipotensão arterial durante anestesia subaracnóidea ${ }^{1}$. O índice de sobrecarga vascular é um previsor de risco cardiovascular em pacientes hipertensos que se correlaciona com a magnitude da redução tensional sistólica durante anestesia geral e após administração de doses indutoras de propofol ${ }^{2,3}$. O índice de pressão sistólica tornozelo/braço, além de predizer a gravidade da doença arterial oclusiva de membros inferiores, também encontra-se diminuído em pacientes hipertensos sem arteriopatia periférica, provavelmente pela alteração da velocidade do fluxo sangüíneo na aorta e nos grandes vasos do membro inferior, causada pelo enrijecimento da parede arterial ${ }^{4}$. Assim, pelo mesmo mecanismo, o índice de sobrecarga vascular e o índice tornozelo/braço de pressão sistólica encontram-se alterados, ainda que em direções 
opostas, em pacientes hipertensos. Assim como o índice de sobrecarga vascular, o índice tornozelo/braço de pressão arterial sistólica correlaciona-se com a magnitude da redução tensional sistólica durante raquianestesia ${ }^{5}$. A hipertensão arterial tem sido relacionada ou não como fator de risco para o desenvolvimento de hipotensão arterial durante anestesias subaracnóideas e peridurais ${ }^{6,7}$.

Este estudo teve como objetivo avaliar diversos fatores pré-anestésicos como previsores independentes de hipotensão arterial durante os primeiros 20 minutos após a injeção subaracnóidea de bupivacaína hiperbárica.

\section{MÉTODO}

Com a aprovação da Comissão de Ética Médica do Hospital Governador Celso Ramos e consentimento verbal, foram estudados prospectivamente 76 pacientes de ambos os sexos, submetidos à anestesia subaracnóidea lombar com bupivacaína a $0,5 \%$ hiperbárica, para cirurgias eletivas. Os pacientes foram monitorizados com monitor pressão arterial oscilométrico não invasivo, cardioscópio (MC5) e oxímetro de pulso. Os seguintes dados foram coletados antes do início da anestesia: idade, sexo, peso, altura, índice de massa corporal (IMC = peso. altura $\left.^{-2}\right)$, história de hipertensão arterial, uso de drogas anti-hipertensivas ou cardio-ativas, medicação pré-anestésica, pressão arterial sistólica (PAS controle $_{\text {e }}$, pressão arterial diastólica $\left(P A D_{\text {controle }}\right)$, freqüência cardíaca $\left(\mathrm{FC}_{\text {controle }}\right)$, saturação periférica da oxi-hemoglobina $\left(\mathrm{SpO}_{2 \text { controle }}\right)$. Também foram medidas, por método oscilométrico, as pressões arteriais sistólica e diastólica no membro inferior, com o manguito localizado logo acima do tornozelo. Todas as medidas de pressão arterial foram realizadas no membro do lado dominante (direito, em todos os pacientes desta amostra). Dos dados hemodinâmicos pré-anestésicos foram calculados: pressão de pulso arterial (PP = PAS PAD), índice de sobrecarga vascular (ISV = 1,33 PAS - 0,33 $P A D$-133), índice tornozelo/braço de pressão arterial sistólica (ITB = PAS membro inferior $_{\text {PAS }} /$ membro superior $)$.

Todos os pacientes receberam, por via venosa, $10 \mathrm{ml} . \mathrm{kg}^{-1} \mathrm{de}$ solução fisiológica antes da realização do bloqueio subaracnóideo.

A medicação pré-anestésica, quando administrada, constou de diazepam $\left(0,15 \mathrm{mg} \cdot \mathrm{kg}^{-1}\right)$ ou midazolam $(0,03$ a 0,05 $\mathrm{mg} \cdot \mathrm{kg}^{-1}$ ) associados ou não a fentanil $1 \mathrm{a} 2 \mathrm{mg} \cdot \mathrm{kg}^{-1}, 5$ a $10 \mathrm{mi}-$ nutos antes da realização da anestesia. As drogas foram administradas a critério do anestesiologista responsável.

As medidas de PAS, PAD e FC foram iniciadas logo após a administração subaracnóidea da dose de bupivacaína a $0,5 \%$ hiperbárica, escolhida pelo anestesiologista responsável (M0) e novas medidas foram realizadas a intervalos de 2,5 minutos até o vigésimo minuto (M2,5 a M20).

Os pacientes receberam oxigênio ( 3 L. $\mathrm{min}^{-1}$ ) durante o período de observação, segundo designado em envelope lacrado e sorteado previamente. Os pacientes não receberam opióides ou benzodiazepínicos durante o período de observação.
Hipotensão arterial foi definida como redução da PAS a valores inferiores a $80 \%$ do nível pré-anestésico ou PAS menor que $90 \mathrm{mmHg}$. Doses de 5 a $10 \mathrm{mg}$ de efedrina foram administradas para tratar tais episódios e oxigênio foi administrado, sob máscara facial, até o retorno da PAS a níveis superiores a $80 \%$ dos pré-anestésicos.

Ao término do período de observação, foram anotados: as doses de bupivacaína e de efedrina, o nível superior do bloqueio sensitivo, pesquisado pelo toque da ponta de uma aguIha e a menor pressão arterial sistólica $\left(\mathrm{PAS}_{\min }\right)$, a partir da qual foi calculado o percentual máximo de redução tensional sistólica $\left(\triangle \mathrm{PAS}=\mathrm{PAS}_{\min }-\mathrm{PAS}\right.$ controle $/ \mathrm{PAS}$ controle $)$.

Os pacientes que não satisfizeram os critérios de hipotensão arterial durante o período de observação formaram o grupo $\mathrm{N}$ e os que apresentaram hipotensão arterial, o grupo $\mathrm{H}$.

Os fatores foram submetidos a análises individuais para comparações entre os grupos, pelos testes $t$ de Student (variáveis contínuas) ou qui-quadrado (variáveis categóricas). As variáveis que tiveram valores de $p$ menor que 0,05 nas análises individuais entraram como variáveis independentes em um modelo de regressão logística. Para facilitar a utilização do modelo logístico, as variáveis significativas, (sexo, idade e nível superior do bloqueio sensitivo) foram dicotomizadas como segue a partir da mediana dos valores da amostra:

a. Sexo : 1 - masculino, 2 - feminino;

b. Idade: 1 - menor que 45 anos, 2 - igual ou maior que 45 anos;

c. Nível superior de bloqueio sensitivo: 1- em ou abaixo de T7, 2 - acima de T7.

Os parâmetros hemodinâmicos de controle, o ISV, o ITB e o $\triangle$ PAS foram comparados, entre os grupos, pelo teste $t$ de Student para amostras independentes.

O nível de significância foi estabelecido em $5 \%$.

\section{RESULTADOS}

Foram excluídos 6 pacientes da amostra, por violações do protocolo do estudo. Dos 70 pacientes restantes, 30 (42,85\%) apresentaram hipotensão arterial e constituíram o grupo $\mathrm{H}$, enquanto os demais formaram o grupo N. Estes grupos diferiram quanto à idade, sexo, peso, altura, nível superior do bloqueio sensitivo e $\triangle$ PAS. Não houve diferenças quanto ao IMC, história de HAS, uso de anti-hipertensivos, de medicação pré-anestésica ou de oxigenioterapia, doses de bupivacaína, parâmetros hemodinâmicos pré-anestésicos, índice de sobrecarga vascular, pressão de pulso arterial ou índice tornozelo/braço de pressão arterial sistólica (Tabelas I, II e III). Foram fatores de previsão independentes de hipotensão arterial: idade igual ou superior a 45 anos, sexo feminino e nível superior do bloqueio sensitivo acima de T7 (Tabela IV). Aaplicação do modelo na amostra possibilitou a classificação correta de $77,5 \%$ dos pacientes do grupo $\mathrm{N}$ e de $76,66 \%$ dos pacientes do grupo $\mathrm{H}$. 
Tabela I - Dados Demográficos

\begin{tabular}{lcc}
\hline & Grupo N & Grupo H \\
\hline Idade $\left(\right.$ anos) $^{1}$ & $40,5 \pm 17,10^{*}$ & $50,66 \pm 16,15$ \\
Sexo $(\mathrm{M} / \mathrm{F})^{2}$ & $31 / 9^{*}$ & $12 / 17$ \\
Peso $(\mathrm{kg})^{1}$ & $74,42 \pm 14,86 *$ & $65,46 \pm 12,45$ \\
Altura $(\mathrm{cm})^{1}$ & $172,95 \pm 8,18^{*}$ & $1,63 \pm 9,3$ \\
IMC $\left(\mathrm{kg} \cdot \mathrm{m}^{-2}\right)$ & $28,80 \pm 4,15$ & $24,60 \pm 4,29$ \\
\hline
\end{tabular}

1 = dados representados pela média $\pm \mathrm{DP} ; 2=$ dados representados pela freqüência; ${ }^{*}=p<0,05$ entre os grupos

Tabela II - Variáveis Peri-Operatórias

\begin{tabular}{|c|c|c|}
\hline & Grupo N & Grupo H \\
\hline História de hipertensão arterial (Sim/Não) ${ }^{1}$ & $6 / 34$ & $7 / 23$ \\
\hline Uso de drogas anti-hiperten- sivas (Sim/Não) ${ }^{1}$ & $5 / 35$ & $4 / 26$ \\
\hline Medicação pré-anestésica (Sim/Não) ${ }^{1}$ & $34 / 6$ & $22 / 8$ \\
\hline Oxigenioterapia per-operatória (Sim/Não) ${ }^{1}$ & $21 / 19$ & $17 / 13$ \\
\hline Dose de bupivacaína $(\mathrm{mg})^{2}$ & $14,5[13 ; 15]$ & $15[13 ; 15]$ \\
\hline Nível superior do bloqueio sensitivo ${ }^{2}$ & Т8 [т6;T8] & $\mathrm{T} 6[\mathrm{~T} 4,5 ; \mathrm{T} 8]^{*}$ \\
\hline Uso de efedrina (Sim/Não) ${ }^{1}$ & $0 / 40$ & $20 / 10$ \\
\hline Dose de efedrina $(\mathrm{mg})^{3}$ & 0 & $12,2 \pm 5,49$ * \\
\hline Máximo percentual de redução da PAS $(\%)^{3}$ & $-8,24 \pm 6,60$ & $-24,71 \pm 8,75^{*}$ \\
\hline
\end{tabular}

$-24,71 \pm 8,75$ *

1 = dados representados pela freqüência; 2 = dados representados pela mediana [quartil superior; inferior]; 3 = dados represent ados pela Média \pm DP; ${ }^{*} p<0,05$ entre os grupos

Tabela III - Variáveis Hemodinâmicas Pré-Anestésicas (Média \pm DP)

\begin{tabular}{lcc}
\hline & Grupo $\mathrm{G}$ & Grupo H \\
\hline PAS - membro superior $(\mathrm{mmHg})$ & $132,57 \pm 18,88$ & $135,23 \pm 22,97$ \\
PAD - membro superior $(\mathrm{mmHg})$ & $79,37 \pm 11,22$ & $81,06 \pm 10,91$ \\
FC - (bpm) & $74 \pm 14,62$ & $77,26 \pm 16,68$ \\
PAS - membro inferior $(\mathrm{mmHg})$ & $152 \pm 23,61$ & $152,96 \pm 23,11$ \\
PAD - membro inferior (mmHg) & $82,25 \pm 21,07$ & $81,66 \pm 11,92$ \\
PP - membro superior (mmHg) & $53,20 \pm 13,07$ & $54,16 \pm 17,03$ \\
PP - membro inferior (mmHg) & $69,75 \pm 21,23$ & $71,30 \pm 20,14$ \\
Índice de sobrecarga vascular & $17,13 \pm 22,53$ & $20,10 \pm 28,10$ \\
Índice tornozelo/braço de PAS & $1,15 \pm 0,12$ & $1,14 \pm 0,16$ \\
\hline
\end{tabular}

Tabela IV - Resumo do Modelo de Regressão Logística

\begin{tabular}{|c|c|c|c|}
\hline & Coeficiente $\beta$ & Razão de chances & Limites de $95 \%$ de confiança \\
\hline Idade $^{1}$ & 1,753 & 5,77 & 1,$56 ; 21,32$ \\
\hline $\operatorname{Sexo}^{2}$ & 2,176 & 8,81 & 2,$04 ; 37,91$ \\
\hline Nível superior do bloqueio sensitivo ${ }^{3}$ & 2,561 & 12,96 & 2,$86 ; 58,65$ \\
\hline Constante $\alpha$ & $-9,922$ & & \\
\hline
\end{tabular}

1. $1:<45$ anos; $2:>45$ anos;

2. 1: masculino; 2 : feminino;

3. 1: igual ou abaixo de T7; 2 : acima de T7

\section{DISCUSSÃO}

Hipotensão arterial durante bloqueio subaracnóideo constitui-se em risco de isquemia miocárdica e cerebral ${ }^{8}$. Por estas razões, o reconhecimento de fatores que predizem maior risco de ocorrência de hipotensão arterial durante bloqueio subaracnóideo, em um dado paciente, pode auxiliar o Anestesiologista a decidir sobre a escolha da técnica ou pela adoção de medidas especiais de monitorização e manuseio farmacológico. A regressão logística permite a construção de mo- delos previsores de probabilidade de ocorrência de um determinado evento, na presença de fatores de risco. Os fatores de risco devem ser de fácil obtenção e conhecidos antes da realização do bloqueio, para que o modelo cumpra sua finalidade preventiva. Neste estudo, dados rotineiramente obtidos durante a consulta pré-anestésica foram testados como potenciais previsores de hipotensão. O período de observação foi limitado aos primeiros 20 minutos após a realização dos bloqueios para evitar que fatores operatórios, como sangramento e mudança de posição, pudessem alte- 
rar a prevalência ou a intensidade da redução tensional sistólica.

A hipertensão arterial tratada não se relaciona com maior prevalência de hipotensão arterial durante bloqueios espinhais ${ }^{9}$. Este estudo confirma tal resultado.

O índice de sobrecarga vascular, um substituto para níveis tensionais arbitrários em estudos epidemiológicos de hipertensão arterial $^{3}$, tem-se correlacionado com a magnitude da redução tensional sistólica em pacientes submetidos à anestesia geral ${ }^{7}$. Em estudo anterior ${ }^{10}$, somente pacientes hipertensos apresentaram correlação significativa entre a redução da pressão arterial e o índice de sobrecarga vascular, após indução de anestesia com propofol. O índice tornozelo/braço de pressão arterial sistólica correlaciona-se com a história de hipertensão arterial $^{2}$ e com a magnitude da redução tensional sistólica após despinçamento da aorta em cirurgias sobre a aorta abdominal ${ }^{6}$. Este índice, tradicionalmente medido com doppler, apresenta valores semelhantes, quando medido pelo método oscilométrico, mais acessível no ambiente cirúrgico, razão pela qual este último foi utilizado ${ }^{5}$. Neste estudo, não houve diferença significativa entre os valores do índice de sobrecarga vascular ou do índice tornozelo/braço de pressão arterial sistólica entre os pacientes que desenvolveram hipotensão arterial e os que não desenvolveram, de forma que não foram testados no modelo logístico.

Assim como em outro estudo ${ }^{1}$, o índice de massa corporal ea administração de benzodiazepínicos associados ou não a opióides antes da realização dos bloqueios não se associaram com a ocorrência de hipotensão arterial. Também não foi demonstrado qualquer efeito preventivo da administração de oxigênio sobre a ocorrência de hipotensão arterial.

Os fatores de previsão independentes de hipotensão arterial identificados neste estudo foram: idade igual ou superior a 45 anos, sexo feminino e nível superior de bloqueio sensitivo acima de $\mathrm{T} 7$.

A idade tem sido relacionada à ocorrência de hipotensão arterial. Diversos fatores fazem com que o idoso apresente maior propensão à hipotensão, como a diminuição da água corporal e menor adaptação do sistema cardiovascular a alterações de postura e volemia ${ }^{11}$.

Em outro estudo ${ }^{12}$, pacientes do sexo feminino apresentaram chance 8,81 vezes maior de desenvolver hipotensão arterial do que os pacientes masculinos.

Neste estudo, o nível superior do bloqueio sensitivo acima de T7 acompanhou-se com chance 13 vezes maior de ocorrência de hipotensão arterial. A extensão do bloqueio simpático, portanto, continua sendo o fator mais fortemente associado com as alterações hemodinâmicas do bloqueio subaracnóideo ${ }^{1}$

O modelo logístico derivado desta amostra mostrou robustez ao classificar corretamente $76,55 \%$ dos pacientes do grupo $\mathrm{He} 77,5 \%$ dos do grupo N. Por isto, a inserção dos códigos das variáveis sexo, idade e nível superior do bloqueio sensitivo na fórmula do modelo:
$\mathrm{P}($ hipotensão $)=1 /\left(1+\mathrm{e}^{-(-9,922+2,176(\text { sexo })+1,753 \text { (idade) }+2,561 \text { (nivel) })}\right)$, pode prever a probabilidade de um dado paciente desenvolver hipotensão arterial sistólica durante os primeiros $20 \mathrm{mi}-$ nutos de anestesia.

\section{Predictors of Early Hypotension During Spinal Anesthesia}

Getúlio Rodrigues de Oliveira Filho, M.D., Jorge Hamilton Soares Garcia, M.D., Ranulfo Goldschimidt, M.D., Adilson José Dal Mago, M.D., Marcos Aguiar Cordeiro, M.D., Felipe Ceccato, M.D.

\section{INTRODUCTION}

The identification of patients with a high risk to develop hypotension during spinal anesthesia has already been object of a study resulting in a logistic model which considered sensory level above $\mathrm{T} 6$ and more than 50 years of age as independent predictors of hypotension during spinal anesthesia ${ }^{1}$. Vascular overload index is a cardiovascular risk predictor in hypertensive patients and is correlated to the magnitude of systolic tensional decrease during general anesthesia and after propofol inducing doses ${ }^{2,3}$. Ankle/arm systolic pressure index, in addition to predicting lower limb oclusive disease severity, is also decreased in hypertensive patients without peripheral arteriopathies, probably due to a change in blood flow velocity in the aorta and lower limb great vessels, caused by the stiffening of the arterial wall ${ }^{4}$. By the same mechanism, vascular overload and ankle/arm systolic pressure indices are changed in hypertensive patients, although in opposite directions. Similar to vascular overload index, ankle/arm systolic pressure index is related to the magnitude of systolic tensional decrease during spinal anesthesia ${ }^{5}$. Hypertension is or not considered a risk factor for hypotension during epidural and spinal anesthesia ${ }^{6,7}$.

This study aimed at evaluating several preanesthetic factors as independent hypotension predictors during the first $20 \mathrm{mi}-$ nutes after spinal hyperbaric bupivacaine.

\section{METHODS}

After the Hospital Governador Celso Ramos Medical Ethics Committee approval and their verbal consent, participated in this prospective study 76 patients of both genders, submitted to elective surgeries under spinal anesthesia with $0.5 \%$ hyperbaric bupivacaine. Patients were monitored with non invasive blood pressure, cardioscope (MC5) and pulse oximetry. The following preanesthetic data were collected: age, gender, weight, height, body mass index $\left(\mathrm{BMI}=\right.$ weight.height $\left.^{-2}\right)$, hypertension history, use of anti-hypertensive or cardioactive drugs, preanesthetic medication, systolic blood pressure $\left(\mathrm{SBP}_{\text {control }}\right)$, diastolic blood pressure $\left(\mathrm{DBP}_{\text {control }}\right)$, heart rate $\left(\mathrm{HR}_{\text {control }}\right)$, peripheral oxyhemoglobin saturation $\left(\mathrm{SpO}_{2 \text { control }}\right)$. 
Lower limb systolic and diastolic blood pressures were also measured by an oscilometric method with the cuff right above the ankle. All blood pressure measurements were performed in the dominant limb (right, for all patients in this sample). From preanesthetic hemodynamic data we calculated: arterial pulse pressure $(\mathrm{PP}=\mathrm{SBP}$ - DBP $)$, vascular overload index $(\mathrm{VOI}=1.33 \mathrm{SBP}-0.33 \mathrm{DBP}-133)$, ankle/arm systolic pressure index $\left(A M I=S B P_{\text {lower limb }} / S^{-1} P_{\text {upper limb }}\right)$.

All patients received $10 \mathrm{ml}^{.} \mathrm{kg}^{-1}$ intravenous saline before spinal blockade.

Premedication, when administered, consisted of diazepam $\left(0.15 \mathrm{mg} . \mathrm{kg}^{-1}\right)$ or midazolam $\left(0.03\right.$ to $\left.0.05 \mathrm{mg} . \mathrm{kg}^{-1}\right)$ associated or not to fentanyl ( 1 to $2 \mathrm{mg}^{\mathrm{kg}}{ }^{-1}$ ) 5 to 10 minutes before anesthesia. Drugs were administered at the sole discretion of the anesthesiologist in charge.

SBP, DBP and HR measurements started soon after $0.5 \%$ hyperbaric bupivacaine spinal injection (M0) and measurements were repeated at 2.5-minute intervals for 20 minutes (M2.5 to M20).

The some patients received ( 3 L. $\mathrm{min}^{-1}$ ) oxygen during the observation period, according to a sealed envelope. Patients did not receive opioids or benzodiazepinics during the observation period.

Arterial hypotension was defined as SBP below $80 \%$ of control or below $90 \mathrm{mmHg}$. Ephedrine (5 to $10 \mathrm{mg}$ ) was administered to treat such episodes and oxygen under facial mask was used until SBP was back to more than $80 \%$ of preanesthetic values.

At the end of the observation period the following parameters were recorded: bupivacaine and ephedrine doses, upper sensory block level, confirmed by pinprick, and lowest systolic blood pressure $\left(\mathrm{SBP}_{\min }\right)$ from which the maximum systolic tensional decrease percentage was calculated $(\triangle \mathrm{SBP}=$ $\left.\mathrm{SBP}_{\min }-\mathrm{SBP}_{\text {control }} / \mathrm{SBP}_{\text {control }}\right)$.
Patients not meeting hypotension criteria during the observation period were allocated to Group $\mathrm{N}$ and those with hypotension to Group H.

Factors were submitted to individual analyses for comparisons between groups by Student's t test (continuous variables) or chi-square test (categorical variables). Variables with $p<0.05$ in individual analyses were considered independent variables in a logistic regression model. To make easier the logistic model, significant variables (gender, age and upper sensory block level) were split in two as from sample values median, as follows:

a. Gender: 1 = male, 2 = female;

b. Age: 1 = less than 45 years, 2 equal to or more than 45 years;

c. Upper sensory block level: 1 = within or below T7, 2 = above T7.

Hemodynamic parameters, VOI, AAI and $\triangle$ SBP were compared between groups by Student's t test for independent samples. Significance level was $5 \%$.

\section{RESULTS}

Six patients were excluded from the study due to protocol noncompliance. From the remaining 70 patients, 30 $(42.85 \%)$ had hypotension and were allocated to group $\mathrm{H}$, while the remaining were allocated to group $\mathrm{N}$. These groups differed in age, gender, weight, height, upper sensory block level and $\triangle$ SBP. There were no differences in BMI, SH, hypertension therapy, preanesthetic medication or oxygen therapy, bupivacaine doses, preanesthetic hemodynamic parameters, vascular overload index, pulse pressure or ankle/arm systolic blood pressure index (Tables I, II and III).

Table I - Demographics Data

\begin{tabular}{|c|c|c|}
\hline & Group N & GroupH \\
\hline Age (years) ${ }^{1}$ & $40.5 \pm 17.10$ * & $50.66 \pm 16.15$ \\
\hline Gender $(\mathrm{M} / \mathrm{F})^{2}$ & $31 / 9$ * & $12 / 17$ \\
\hline Weight (kg) ${ }^{1}$ & $74.42 \pm 14.86$ * & $65.46 \pm 12.45$ \\
\hline Height $(\mathrm{cm})^{1}$ & $172.95 \pm 8.18$ * & $1.63 \pm 9.3$ \\
\hline $\mathrm{BMI}\left(\mathrm{kg} \cdot \mathrm{m}^{-2}\right)$ & $28.80 \pm 4.15$ & $24.60 \pm 4.29$ \\
\hline
\end{tabular}

$1=$ Data expressed in Mean $\pm S D ; 2=$ Data represented by frequency; ${ }^{*}=p<0.05$ between groups

Table II - Perioperative Variables

\begin{tabular}{|c|c|c|}
\hline & Group N & Group H \\
\hline Hypertension history (Yes/No) ${ }^{1}$ & $6 / 34$ & $7 / 23$ \\
\hline Use of anti-hypertensive drugs (Yes/No) ${ }^{1}$ & $5 / 35$ & $4 / 26$ \\
\hline Preanesthetic medication (Yes/No) ${ }^{1}$ & $34 / 6$ & $22 / 8$ \\
\hline Perioperative oxygen therapy (Yes/No) ${ }^{1}$ & $21 / 19$ & $17 / 13$ \\
\hline Bupivacaine dose $(\mathrm{mg})^{2}$ & $14.5[13 ; 15]$ & $15[13 ; 15]$ \\
\hline Upper sensory block ${ }^{2}$ & T8 [T6;T8] & $\mathrm{T} 6[\mathrm{~T} 4.5 ; \mathrm{T} 8]$ * \\
\hline Use of ephedrine (Yes/No $)^{1}$ & $0 / 40$ & $20 / 10$ \\
\hline Ephedrine dose $(\mathrm{mg})^{3}$ & 0 & $12.2 \pm 5.49$ * \\
\hline Maximum SBP decrease (\%) ${ }^{3}$ & $-8.24 \pm 6.60$ & $-24.71 \pm 8.75^{*}$ \\
\hline
\end{tabular}

1 = Data represented by frequency; 2 = Data represented by median (upper. lower quartile); $3=$ Data represented in Mean \pm SD; * $p<0.05$ between groups 
Table III - Preanesthetic Hemodynamic Variables (Mean \pm SD)

\begin{tabular}{lcc}
\hline & Group N & Group H \\
\hline SBP - upper limb $(\mathrm{mmHg})$ & $132.57 \pm 18.88$ & $135.23 \pm 22.97$ \\
DBP - upper limb $(\mathrm{mmHg})$ & $79.37 \pm 11.22$ & $81.06 \pm 10.91$ \\
HR - (bpm) & $74 \pm 14.62$ & $77.26 \pm 16.68$ \\
SBP - lower limb $(\mathrm{mmHg})$ & $152 \pm 23.61$ & $152.96 \pm 23.11$ \\
DBP - lower limb $(\mathrm{mmHg})$ & $82.25 \pm 21.07$ & $81.66 \pm 11.92$ \\
PP - upper limb $(\mathrm{mmHg})$ & $53.20 \pm 13.07$ & $54.16 \pm 17.03$ \\
PP - lower limb $(\mathrm{mmHg})$ & $69.75 \pm 21.23$ & $71.30 \pm 20.14$ \\
Vascular overload index & $17.13 \pm 22.53$ & $20.10 \pm 28.10$ \\
Ankle/arm SBP index & $1.15 \pm 0.12$ & $1.14 \pm 0.16$ \\
\hline
\end{tabular}

Table IV - Summary of Logistic Regression Model

\begin{tabular}{lccc}
\hline & Coefficient $\beta$ & Odds Ratio & Confidence Limits of 95\% \\
\hline Age $^{1}$ & 1.753 & 5.77 & $1.56 ; 21.32$ \\
Gender $^{2}$ & 2.176 & 8.81 & $2.04 ; 37.91$ \\
Upper sensory block level $^{3}$ & 2.561 & 12.96 & $2.86 ; 58.65$ \\
Constant $\alpha$ & -9.922 & & \\
\hline
\end{tabular}

1. $1:<45$ years; $2: \geq 45$ years;

2. 1: male; 2 : female;

3. 1: equal to or below T7; 2 : above T7

Factors considered as independent hypotension predictors were: age equal to or above 45 years, female gender and upper sensory block level above T7 (Table IV). The model applied to the sample allowed for $77.5 \%$ of accurate classification of patients in Group $\mathrm{N}$ and $76.66 \%$ in Group $\mathrm{H}$.

\section{DISCUSSION}

Hypotension during spinal block poses a risk for myocardial and brain ischemia ${ }^{8}$. So, the identification of predicting factors for a higher hypotension risk during spinal anesthesia may help the anesthesiologist in his decision on the technique or on special monitoring measures and drug handling. Logistic regression methods enable the building of predicting models of the probability of a certain event to occur in the presence of risk factors. Risk factors must be easily obtained and known before the blockade for the model to meet its preventive aim. In our study, routine data obtained during preanesthetic evaluations were tested as potential hypotension predictors. The observation period was limited to 20 minutes after blockade to prevent perioperative factors, such as bleeding and change in position, to affect the prevalence or intensity of systolic tensional decrease.

Treated hypertension is not related to a higher hypotension prevalence during spinal blocks ${ }^{9}$ and this was confirmed by our study.

Vascular overload index, an alternative for arbitrary tensional levels in hypertension epidemiological studies ${ }^{3}$, is related to the magnitude of systolic tensional decrease in patients submitted to general anesthesia ${ }^{7}$. In a previous study ${ }^{10}$, only hypertensive patients had a significant correlation between blood pressure decrease and vascular overload index after propofol-induced anesthesia. Ankle/arm systolic blood pressure index is correlated to previous hypertension ${ }^{2}$ and to the magnitude of systolic tensional decrease after aorta clamping release in abdominal aorta surgeries ${ }^{6}$. Such index, traditionally measured with doppler, has similar values when measured by the oscilometric method which is more widely available in the operating room, and that is why this was our method of choice ${ }^{5}$. There have been no significant differences in vascular overload index or ankle/arm systolic blood pressure index between patients developing hypotension and normotensive patients, so they were not tested in the logistic model.

As in a different study ${ }^{1}$, body mass index and pre-blockade administration of benzodiazepinics, associated or not to opioids, were not associated to hypotension. It was also not shown any preventive effect of oxygen administration on hypotension occurrence.

Independent hypotension predictors identified in our study were: age equal to or above 45 years, female gender and upper sensory block above T7.

Age has been related to hypotension. Several factors lead elderly people to be more prone to hypotension, such as a decrease in body water and poorer adaptation of the cardiovascular system to posture and volume changes ${ }^{11}$.

In other study ${ }^{12}$, female patients had 8.81 more chances of developing hypotension than males.

In our study, upper sensory block above T7 had 13 times more chances of developing hypotension. Sympathetic block extension, then, is still the factor more strongly related to spinal block hemodynamic changes ${ }^{1}$.

The logistic model derived from our sample was robust in terms of accurately classifying $76.55 \%$ of Group $\mathrm{H}$ patients and $77.5 \%$ of Group N patients. So, the insertion of gender, age and upper sensory block level codes in the model's formula: 
$\mathrm{P}($ hypotension $)=1 /\left(1+\mathrm{e}^{-(-9.922+2.176 \text { (gender })=1.753(\text { age })+(2.561(\text { level }))}\right)$, may predict the probability of a given patient to develop systolic hypotension during the first 20 minutes after anesthesia.

\section{REFERÊNCIAS - REFERENCES}

01. Tarkkila P, Isola J - A regression model for identifying patients at high risk of hypotension, bradicardia and nausea during spinal anesthesia. Acta Anaesthesiol Scand, 1992;36:554-558.

02. Hugue CJ, Safar ME, Aliefierakis MC - The ratio between ankle and brachial systolic pressure in patients with sustained uncomplicated essential hypertension. Clin Sci, 1988;74:179-182.

03. Franklin SS, Weber MA - Measuring hypertensive cardiovascular risk: the vascular overload concept. Am Heart J, 1994;128: 793-803.

04. Kelly R, Hayward C, Avolio A et al - Noninvasive determination of age-related changes in the human arterial pulse. Circulation, 1989;80:1652-1659.

05. Adiseshiah M, Cross FW, Belsham PA - Ankle blood pressure measures by automatic oscillotonometry: a comparison with Doppler pressure measurements. Ann R Coll Surg Engl, 1987;69:271-273

06. Vretzakis G, Koutsias S, Grosomanidis V - A simple preoperative indicator of the haemodynamic changes during surgery of the abdominal aorta. Br J Anaesth, 1997;78(Suppl 1):A118.

07. Young C, Hilton A, Silbert K et al - Vascular overload index predicts intraoperative hypotension. Anesth Analg, 1996;82:S507.

08. Juelsgaard P, Sand NP, Felsby S et al - Perioperative myocardical ischaemia in patients undergoing surgery for fractured hip randomized to incremental spinal, single-dose spinal or general anaesthesia. Eur J Anaesthesiol, 1998;15:656-663.

09. Dagnino J, Prys-Roberts C - Studies of anaesthesia in relation to hypertension. VI: cardiovascular responses to extradural blockade of treated and untreated hypertensive patients. $\mathrm{Br} J$ Anaesth, 1984;56:1065-1073.

10. Oliveira Filho GR, Teixeira Filho N, Pederneiras SG et al - Índice de sobrecarga vascular e pressão arterial como indicadores de variação tensional sistólica após indução anestésica com propofol em pacientes normotensos e hipertensos. Rev Bras Anestesiol, 2000;50:269-272.

11. Critchley LA, Stuart JC, Short TG et al - Haemodynamic effects of subarachnoid block in elderly patients. $\mathrm{Br} J$ Anaesth, 1994;74:464-470.

12. Tarkkila PJ, Kaukinen S - Complications during spinal anesthesia: a prospective study. Reg Anesth, 1991;16:101-106.

\section{RESUMEN}

Oliveira Filho GR, Garcia JHS, Goldschimidt R, Dal Mago AJ, Cordeiro MA, Ceccato F - Factores de Previsión de Hipotensión Arterial Precoz en Anestesia Subaracnóidea

Justificativa y Objetivos - La identificación de factores de previsión de hipotensión arterial durante bloqueos subaracnóideos puede interferir en la selección de la técnica o en la utilización de medidas preventivas. Este estudio evaluó factores pré-anestésicos como previsores independientes de hipotensión arterial durante bloqueo subaracnóideo.

Método - Fueron estudiados 76 pacientes de ambos sexos, sometidos a anestesia subaracnóidea con bupivacaína $0,5 \%$ hiperbárica. Fueron colectados: edad, sexo, peso, altura, índice de masa corporal, historia de hipertensión arterial, uso de drogas anti-hipertensivas, medicación pré-anestésica, presión arterial sistólica (PAS) y diastólica (PAD) en el brazo y en el tobillo, frecuencia cardíaca (FC), presión de pulso arterial, índice de sobrecarga vascular, índice tobillo/brazo de PAS, dosis de bupivacaína, nivel superior del bloqueo sensitivo, y la menor PAS, medida a intervalos de 2,5 minutos hasta el vigésimo minuto. Oxigeno a $3 \mathrm{~L}$. $\mathrm{min}^{-1}$ fue administrado, según sorteo. Hipotensión arterial fue definida como reducción de la PAS a valores inferiores a $80 \%$ del nivel pré-anestésico o PAS menor que $90 \mathrm{mmHg}$. Fue utilizada regresión logística para identificar las variables asociadas con la ocurrencia de hipotensión arterial.

Resultados - Fueron factores de previsión de hipotensión arterial: edad mayor que 45 años, sexo femenino y nivel superior del bloqueo sensitivo arriba de T7.

Conclusiones - Fueron identificados factores de previsión independientes de reducción tensional sistólica arriba de $20 \%$ de los valores pré-anestésicos la edad superior de 45 años, el sexo femenino y el nivel superior del bloqueo sensitivo arriba de $\mathrm{T} 7$. 